\title{
El tesauro de infraestructura cultural universitaria: investigación y metodología
}

\author{
Catalina Naumis-Peña*
}

Artículo recibido:

7 de diciembre de 2019

Artículo aceptado:

30 de marzo de 2020

Artículo de investigación

\section{Resumen}

Se explica la metodología de investigación-acción para conformar un tesauro sobre infraestructura cultural universitaria en el marco de un proyecto institucional que promueve la investigación universitaria, la difusión de sus resultados y el aprendizaje de los alumnos. Un tesauro es el resultado de una investigación de los significados de los términos en un ámbito específico para fomentar el entendimiento entre los usuarios finales de un sistema de información. La metodología desarrollada permitió obtener un tesauro y fomentar el aprendizaje de un grupo interdisciplinario de alumnos para cumplir con los objetivos del proyecto respaldado por la Universidad Nacional Autónoma de México.

* Instituto de Investigaciones Bibliotecológicas y de la Información, UNAM, México naumis@unam.mx_cnaumis@gmail.com

INVESTIGACIÓN BIBLIOTECOLÓGICA, vol. 34, núm. 85, octubre/diciembre, 2020, México, ISSN: 2448-8321 pp. 137-153 
Palabras clave: Árboles de Dominio; Terminología de Infraestructura Cultural; Comunicación Universitaria; Diseño de la Estructura Tesaural

The University Cultural Infrastructure Thesaurus: research and methodology Catalina Naumis-Peña

\section{Abstract}

The action research methodology followed to form a thesaurus on university cultural infrastructure within the framework of an institutional project that encourages university research, dissemination of its results and student learning is explained. A thesaurus is the result of an investigation about the meanings of the terms in a specific area to foster understanding among the end users of an information system. The methodology developed allowed to achieve the objective of obtaining a thesaurus and encouraging the learning of an interdisciplinary group of students to meet the objectives of the project supported by the National Autonomous University of Mexico.

Keywords: Domain Trees; Cultural Infrastructure Terminology; University Communication; Thesaurus Structure Design

\section{INTRODUCCIÓN}

$\mathrm{E}^{1}$ 1 objetivo del trabajo es presentar la investigación y metodología para obtener un tesauro sobre la infraestructura cultural universitaria (ICU), con la finalidad de implementarlo en la organización temática de un sistema de información que facilite la comunicación entre programadores de actividades culturales en una organización universitaria de grandes dimensiones: la Universidad Nacional Autónoma de México (UNAM) (https://www.cultura.unam.mx/).

En primera instancia cabe señalar que la infraestructura se define conceptualmente como la base que sustenta, soporta o sostiene una organización 
mediante un conjunto de elementos o servicios que son necesarios o considerados importantes para la creación o producción y marcha de una organización (RAE, 2019). La investigación de los términos significativos del ámbito a organizar busca la representación de sus fenómenos, reconociendo en ello la tendencia sociocognitiva de la terminología (Hjørland, 2002b).

De esta manera, la creación del tesauro se refiere a una esfera específica en un medio universitario, en un idioma que es el español, con entidades concretas, visibles y tangibles que designan espacios y recursos técnicos; en suma, se trata de un instrumento lingüístico de organización de un ámbito con características sociales y terminológicas que lo hacen diferente a cualquier otro tesauro que se haya podido localizar.

La clave de una comunicación adecuada en un sistema de información es que el emisor y el receptor compartan no solamente las mismas palabras, sino que también les asignen el mismo significado evitando la confusión (Naumis Peña, Rodríguez García y Guadarrama Sánchez, 2019: 2). "El tesauro se define como una estructura lingüística de clasificación temática o facetada cuya organización básica está conformada por una relación de descriptores que representan o describen autoridades o contenidos semánticos por medio de unidades lingüísticas extraídas del lenguaje de una disciplina o área específica del conocimiento" (Naumis Peña, 2000: 104).

La conformación del sistema conceptual de los descriptores tesaurales se realiza a través de las relaciones jerárquicas, asociativas y equivalentes, con los siguientes resultados de la contextualización: desambiguación de los términos polisémicos, asistencia para la navegación al ubicar temas generales y específicos, efectividad en las estrategias de recuperación de información y posibilidades de aplicación en mecanismos de inteligencia artificial (Naumis Peña, 2003: 37; Clavier, 2019: 616).

El propósito es establecer términos bien formulados y estrictos que puedan usarse para producir las frases de indización del sistema de información (Fugmann, 1992; 1993). Desarrollar un sistema clasificatorio supone conformar una estructura de conocimiento y no es suficiente saber sobre las cosas, más bien, definir la relación de una cosa con la otra es lo que sirve y determina la comprensión más profunda (Kwaśnik, 2019: 657).

En este caso, la estructura de conocimiento que se propone es el tesauro sobre la ICU-UNAM integrado por el conjunto de espacios culturales (bienes inmuebles) complementados con recursos técnicos (bienes muebles) en donde se realizan actividades como la danza, las representaciones teatrales, las proyecciones cinematográficas, las conferencias, los conciertos, las muestras artísticas, etc. La denominada ICU, a diferencia de los otros bienes muebles e inmuebles que se encuentran en una universidad, se relaciona con la 
potencial utilidad para programar actividades culturales ya sean externas o internas y únicamente se refiere a ocupación de espacios, tiempos específicos de ocupación, equipos complementarios para eventos y datos sobre la administración de los espacios y los recursos humanos disponibles necesarios o considerados necesarios para realizarlos.

Los espacios culturales universitarios son de los siguientes tipos: construidos especialmente para desarrollar ciertas actividades culturales, adaptados a funciones no programadas en su construcción, recintos multiusos, o áreas donde se pueden programar eventos culturales como estadios, explanadas, plazas de acceso a conjuntos de edificaciones, patios, etc.

Es decir, que se reconoce una primera división entre tipos de espacios profesionales y no profesionales que se presentan en la universidad y que condicionan de alguna manera las actividades que se llevan a cabo en su interior. Por lo tanto, es posible encontrar recintos especializados (profesionales) que fueron construidos a propósito para realizar una actividad artística específica como el baile o los conciertos, y espacios no especializados que se edificaron para responder primordialmente a las actividades académicas de tipo cultural que se llevan a cabo en la universidad, como conferencias, mesas redondas, foros académicos, ceremonias de premiación, etc. y que también son susceptibles para programar e integrar el vocabulario a desarrollar.

Las áreas en donde se presentan las actividades y expresiones culturales poseen diversos componentes espaciales, mecánicos, eléctricos y electrónicos orientados a la creación de atmósferas y dinámicas de los eventos culturales que se presenten en ellos. En la comunicación entre los diversos usuarios universitarios de recintos y áreas culturales es fundamental usar las designaciones terminológicas comprensibles para los organizadores y participantes en los eventos a realizar, con la finalidad de evitar los errores propios de la comunicación humana (Naumis Peña, Rodríguez García y Guadarrama Sánchez, 2019: 2). "El propósito de un tesauro es guiar al indizador y al buscador para seleccionar los mismos términos preferidos o combinar los términos preferidos para representar un tema asignado" (ISO 25964-1, 2011: 12).

Se consideran como los principales usuarios del tesauro insertado como elemento constitutivo del proyecto de sistema de información a las personas involucradas en el trabajo cultural de la Coordinación de Difusión Cultural (CDC) de la UNAM, el área de Planeación del Subsistema de Actividades Culturales, así como otros responsables en el Subsistema y los coordinadores de actividades culturales en diferentes sedes, instancias y sitios de educación media superior y superior, centros e institutos de investigación. Esta herramienta permite que la universidad se fortalezca en el campo de la difusión cultural al registrar, interrelacionar y facilitar la consulta de sus recursos sobre el tema. 
La programación efectiva y la difusión de las actividades culturales de la universidad requieren conocer sus posibilidades y sirven como historial y protocolo a los responsables de eventos culturales, que usan o administran de manera permanente o esporádica alguna infraestructura cultural. De ahí la necesidad de sistematizar las funciones, atributos e instrumentos sobre la infraestructura disponible, lo que da lugar al refuerzo del crecimiento de nuevas formas de difusión cultural.

Los objetivos de la universidad son tres: docencia, investigación y difusión de la cultura. Los tres son atendidos en cada uno de los campus de la universidad por separado. Además, existe una infraestructura cultural central administrativa, que no sólo sirve a la universidad, sino también a la población interesada en los eventos que promueve. La CDC es responsable de este segmento y, a menudo, apoya actividades similares en las diferentes dependencias universitarias.

Los espacios culturales que posee la universidad son múltiples, en diversos lugares de la Ciudad de México, los estados e incluso en el extranjero. La universidad posee además un área especializada, el Centro Cultural Universitario (CCU), la cual "Se trata de una obra arquitectónica sin precedente alguno en el ámbito cultural universitario y nacional, en la que cada una de las manifestaciones artísticas cuentan con espacios idóneos para la realización de eventos sociales y empresariales: conciertos, presentaciones, grabación de discos, conferencias y mesas redondas" (Wikipedia, 2019).

En el CCU se encuentra desde un espacio escultórico hasta una de las mejores salas de conciertos a nivel latinoamericano, la Sala Netzahualcoyotl; están asimismo la Sala Miguel Covarrubias para danza, el teatro Juan Ruiz de Alarcón, el Foro Sor Juana Inés de la Cruz o la Sala Carlos Chávez, una pequeña sala de música de cámara; las salas de cine Julio Bracho, José Revueltas y Carlos Monsiváis, el Centro Universitario de Teatro, a los que se suman la Biblioteca Nacional y la Hemeroteca Nacional. Como dato histórico, el complejo arquitectónico fue construido entre 1976 y 1980, complementado con el Museo Universitario de Arte Contemporáneo terminado en 2008. En los años intermedios se agregaron una cafetería y una librería (Wikipedia, 2019).

La UNAM dispone de una estructura arquitectónica y de recursos técnicos en materia cultural que responden en buena medida al liderazgo ejercido en el movimiento cultural mexicano. 
El tesauro de la ICU-UNAM fue desarrollado con el método investigación-acción. Baskerville y Wood-Harper (1996) lo describen como un acercamiento desde la investigación científica, basado en diagnósticos, intervenciones y generación de aprendizajes, para construir un modelo cíclico que consta de cinco fases: diagnóstico, modelo metodológico, intervención e implementación, evaluación, y formulación de aprendizajes.

Se consideró viable la utilización de este método porque además de la investigación incluye la formulación de aprendizajes que, en el PAPIIT, mecanismo de apoyo universitario para crear este sistema de información, son elementos fundamentales para obtener el financiamiento. ${ }^{1}$

\section{Diagnóstico}

En la primera fase de diagnóstico o planificación se establecieron los elementos que se utilizarían para la modelización de la estructura del tesauro, a través de la consulta a los miembros de la CDC participantes en el proyecto y la propia base de datos que se desarrollaba en paralelo.

Para conformar la base de datos en la CDC se tipificaron los espacios culturales mediante visitas técnicas de exploración a los recintos de la UNAM y la consulta de sistemas de información cultural de otros ámbitos. Se identificaron los espacios en los que se llevan a cabo las actividades culturales en cada entidad académica y se diseñó un cuestionario para levantar la información en los recintos universitarios. En el diseño del proyecto y modelado del tesauro se aprovecharon aspectos recuperados para armar la propuesta de base de datos desarrollada por la CDC y los resultados de la exploración, apoyada además por una intensa búsqueda documental y el análisis de los contenidos expuestos en los resultados de los cuestionarios aplicados a los responsables de los espacios.

El análisis que supuso esta primera etapa está basado en los principios de garantía para conformar la esfera específica de conocimiento: la garantía literaria, bibliográfica o documental, la garantía del usuario, la garantía organizacional y la cultural (Martínez Tamayo y Mendes, 2015: 94; Barité, 2019: 651). Los términos para recopilar e integrar el tesauro cumpliendo los estándares de las garantías recomendadas por los especialistas se extraen: 
- De la literatura sobre el tema. La investigación documental permitió contar con una considerable cantidad de diccionarios, glosarios, enciclopedias, vocabularios controlados, tesauros de arte y arquitectura, trabajos académicos universitarios sobre espacios culturales específicos y generales, videos e imágenes acerca de los temas referidos;

- De los usos por los destinatarios del sistema de información porque representan significados claros para los involucrados. Se consultaron a los usuarios finales a través de entrevistas y un representante del grupo destinatario participó como miembro del equipo de trabajo;

- De los elementos que representan los usos en la organización, en este caso las características del lenguaje usado en la universidad a través de las entrevistas y la aplicación de los cuestionarios usados para desarrollar el sistema de información, y

- De la garantía cultural, respetando las particularidades de la comunidad académica y usando los mismos instrumentos que en la garantía organizacional porque en la universidad están muy ligadas estas dos garantías.

\section{Modelo metodológico}

Sin duda, en el conocimiento obtenido en esta primera etapa se reflejaron los tipos de recintos y su caracterización a través de la descripción de los componentes y elementos, los atributos, los recursos técnicos, el aforo, los acabados, las dimensiones generales, etc., además de las áreas en las que se encuentran segmentados para su gestión y uso.

A partir de ese conocimiento reflejado en la base de datos se definieron las tipologías que conforman la ICU-UNAM para modelar una aproximación al ámbito de actividad, y se obtuvo una primera categorización tipificando los espacios y los recursos técnicos para desarrollar los árboles de dominio (Hjørland, 2002a; Smiraglia, 2012) por grupos separados, que constituyen el núcleo de categorías principales del modelo inicial de tesauro propuesto:

auditorio, biblioteca, cine, explanada, foro al aire libre, museo, sala de conciertos, sala de conferencias, sala de proyección audiovisual, sala de usos múltiples, salón de danza, salón de música, teatro

Una vez definida la esfera de conocimiento, se determinó una primera aproximación en las temáticas tratadas para asegurarse de que no existieran tesauros documentales factibles de ser utilizados para satisfacer los requerimientos del sistema de información que sustentarían, se adquirió mayor claridad acerca 
del dominio en el que debe operar el tesauro y en el que se debería ahondar para extraer la terminología que lo conforma; por lo tanto, como resultado de esta segunda etapa completada, se obtuvo el primer acercamiento con un modelo inicial del tesauro y mayor claridad en el dominio a estudiar: "...thesaurus was on the agenda, but the design was to be based on the results of the domain study" (Lykke, 2001: 774).

\section{Intervención e implementación}

Luego de clasificados los tipos de espacios y los componentes que los caracterizan, utilizando ese modelado terminológico inicial se conformó un grupo de trabajo con el investigador que participaba en el proyecto a través de la propuesta de metadatos para la base de datos. Se incorporaron además los estudiantes incluidos en el proyecto, a los que se fue introduciendo en la dinámica de la construcción del tesauro: dos tesistas de Arquitectura, un estudiante de doctorado en Bibliotecología, y tres estudiantes del séptimo semestre de la licenciatura en Bibliotecología. Las reuniones semanales eran complementadas con la asistencia de un representante de la CDC que informaba las características de la base de datos donde se insertaría el tesauro y un informático que asesoraría en la evaluación del sistema de información.

Un estudiante de Arquitectura y el de doctorado en Bibliotecología diseñaron una plantilla de trabajo mediante los servicios web para la recopilación de los términos por las categorías principales propuestas. Se distribuyeron los temas a los estudiantes participantes, quienes en reuniones semanales iban presentando sus avances producto del análisis y la confrontación entre diferentes obras en la literatura especializada. Por cada término se discutían la validez, las jerarquizaciones, las relaciones y las definiciones que se recopilaban en la literatura.

\section{Evaluación}

La observación del trabajo realizado por los alumnos durante sus presentaciones servía para explicar las lagunas o inconsistencias en el desarrollo de cada uno de los temas y sin duda para ahondar en el dominio de conocimiento. La construcción de una estructura arborescente era el problema mayor por las limitaciones de la hoja de cálculo, puesto que cada término tiene una celda y el crecimiento de columnas a veces dificultaba profundizar en los términos jerarquizados. Después de cada presentación se hacían las correcciones y los archivos se subían a Google Drive para observar y continuar con la evaluación. 
Mientras tanto, se hizo una evaluación de software de tesauros partiendo de los recomendados en la literatura y se decidió optar por uno de bajo costo, en lugar de los de acceso libre que no resolvían la funcionalidad del tesauro que incluye una estructura de conocimiento en la que se basa el sistema de información (Campos et al., 2006).

El software es imprescindible y debe evaluarse su funcionalidad, costos y apoyo metodológico, entre otras características. El software se adquirió con sólo cinco licencias alojadas en computadoras aisladas debido al alto costo que suponía comprarlo con la opción de trabajar en red. Al mismo tiempo, se subían los listados al software para dominarlo y facilitar las presentaciones que se iban haciendo por separado, es decir, por cada una de las categorías. Sin duda, la categoría con la mayor dificultad y con el mayor número de sesiones de discusión fue la de teatro.

El lenguaje coadyuva a las diversas aristas disciplinarias y administrativas que se relacionan en dicho contexto para obtener un mejor entendimiento y comprensión acerca de cada elemento que se implementa en el teatro, como pueden ser las divisiones entre las áreas del público y las interiores, en donde interactúan músicos, cantantes, actores, tramoyistas o maquinistas, carpinteros, escenógrafos, utileros, vestuaristas, entre otras actividades, cada uno de ellos comunicándose entre sí, a veces con confusiones e imprecisiones para el conjunto de los profesionales y técnicos que conviven día a día (Theatre Projects Consultants, 2016:2).

Se consultó cada término relacionado con teatro en el Art \& Architecture Thesaurus (ATT), desarrollado por el Getty Research Institute (2019), tanto la versión en inglés como la traducción al español. Las equivalencias en el español del AAT con las usadas por la comunidad de hablantes del ámbito de difusión cultural universitario para referirse a los aspectos y características del teatro en México no son comprensibles en todos los casos, basta mencionar que la designación que en México se conoce como "sala del teatro" en el AAT se anota como "espacio de auditorio" (Naumis Peña, Rodríguez García y Guadarrama Sánchez, 2019:3-4).

En los planteles de enseñanza se generaliza como espacio cultural al auditorio. Si el término utilizado en los recintos profesionales de teatro es "espacio de auditorio" se confunde la comunicación. De esta manera, no quedará especificada la conformación de la sala de teatro al interior del teatro y se presentaría en la misma categoría del espacio de auditorio, que es mucho más general. Por ejemplo, un espacio de auditorio puede comprender el lugar al aire libre donde se colocan sillas para comodidad de los asistentes. Una sala es un término con significado más específico referido al interior de los recintos. 
El AAT es abundante en términos relacionados con los teatros al aire libre e incluso al teatro griego, pero carece de otras especificaciones relacionadas con los recursos técnicos del teatro. En el ámbito universitario que se representa no son frecuentes los teatros al aire libre y los recursos técnicos son fundamentales (Naumis Peña, Rodríguez García y Guadarrama Sánchez, 2019: 5).

Una vez que se recopilaron la generalidad de los términos y sus definiciones fueron agrupados bajo teatro con las siguientes subcategorías: área pública externa, área pública interna, área escénica, área administrativa y área técnica, ello con el fin de poder delimitar el universo del teatro de acuerdo a sus conjuntos y sus respectivos elementos para establecer una estructura jerarquizada del espacio teatral, con la idea de la actividad siguiente de inserción en el tesauro de las relaciones de equivalencia o sinonimia, las asociativas entre descriptores que expresan cierta afinidad semántica entre conceptos distintos y las definiciones que aclaran términos. Las relaciones, como se puede observar, funcionan en un lugar y tiempo particular; hay muchas formas de interpretar, visualizar explicar los fenómenos porque el valor de cualquier enfoque depende de muchos factores, incluido el contexto (Kwaśnik, 2019: 658).

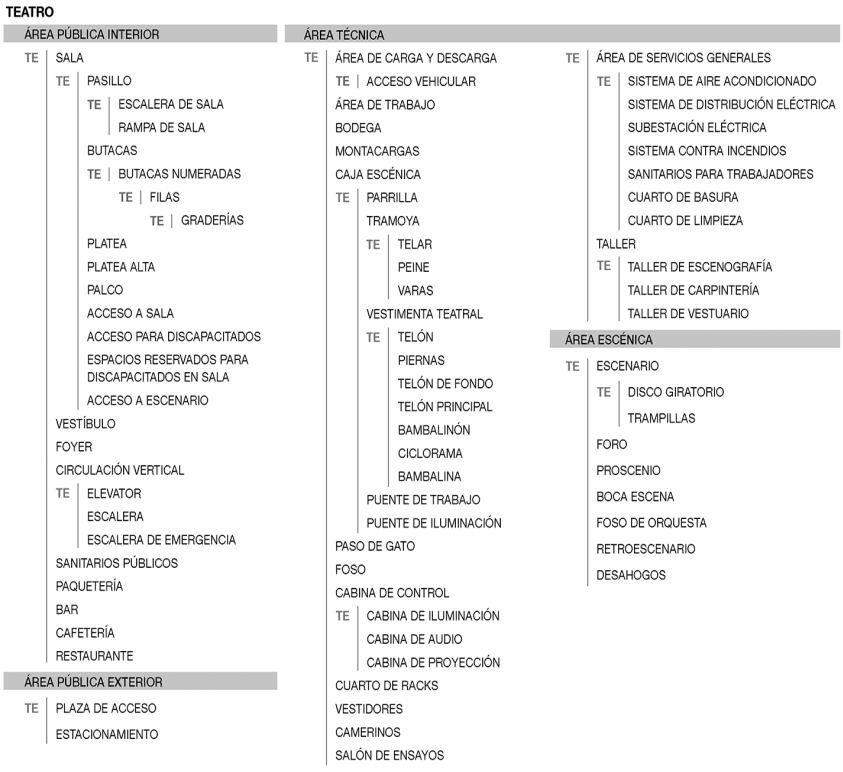

Figura 1. Propuesta del árbol de dominio sobre teatro Fuente: adaptación Luis E. Sánchez 


\section{FORMULACIÓN DE APRENDIZAJES Y ANÁLISIS}

DE LOS RESULTADOS CON EL EQUIPO

Como se puede observar en la Figura 1, el árbol de dominio está dividido en áreas para presentar los términos del teatro en el lugar donde se localizan. En principio se creó un área externa y otra interna, pero fue cambiando a medida que se razonaban los espacio y los términos que los representaban.

Una decisión del grupo de trabajo fue designar el área exterior como ÁREA PÚBLICA EXTERIOR para diferenciarla del ÁREA DE CARGA Y DESCARGA, y al observar su relación con la BODEGA, MONTACARGAS y caja escénica se decidió agruparla en un ÁREA TÉCNICA.

Por ejemplo, un término como VESTIDORES ocupa dos espacios: lugar donde se agrupan las vestimentas utilizadas en las diferentes presentaciones llevadas a cabo (a diferencia de los CAMERINOS que son más privados); el VESTIDOR DE LOS TRABAJADORES que se incluye bajo la categoría del ÁREA DE TRABAJADORES (para distinguirlo se le agregó el tipo de vestidor). Además, existe vestimenta como la VESTIMENTA TEATRAL que no está relacionada con la que usan los actores, sino que es del propio teatro y se ubica en el escenario y los telones.

El equipo de trabajo estudiaba antes de cada sesión para entender cómo funcionaba el espacio y representarlo en el árbol de dominio, ocasionando movimientos constantes en el mismo.

\section{Discusión DE LOS RESULTADOS}

La utilización de la jerarquía supone el acomodo una y otra vez de los términos para tomar decisiones acerca de cuál es el mejor lugar para transformarlo y ubicarlo como descriptor, de modo que no se repita bajo otras jerarquías. Sin embargo, es fundamental no separar el descriptor de las categorías que lo distinguen y relacionarlo con esa otra categoría o subcategoría en la que no quedó jerarquizado para que el contexto exprese las relaciones de significado que lo definen (Jacob, 2004: 89).

Entre las relaciones que definen el contexto de un descriptor en el tesauro está la asociativa, que ofrece la posibilidad de que en diferentes categorías, subcategorías o clases se incluya como asociado ese término que podría haber sido jerarquizado. Es decir, a través del vocabulario se relaciona semánticamente, de una u otra manera, el descriptor, y se le asigna un lugar que lo explica. "El contexto define el alcance y el vocabulario. Decide sobre los elementos mismos y qué relaciones clasificatorias son pertinentes" (Kwaśnik, 2019: 663). 
Por ejemplo, el descriptor ACCESO PARA DISCAPACITADOS que se repite una y otra vez en las diferentes subcategorías o áreas debe aparecer una sola vez en el árbol de dominio. Lo importante es captar ese término y luego ubicarlo en el tesauro relacionado en diferentes posiciones.

El análisis del término en cuestión mostró que existen facilidades para discapacitados en el ÁREA PÚBLICA EXTERIOR y en el ÁREA PÚBLICA INTERIOR, y dentro de ésta en el VESTÍBULO, en la SALA o para subir al ESCENARIO. En la Figura 2 se observa como TE (Término Específico) de ACCESO A SALA, pero también aparece como término relacionado en ACCESO PÚBLICO EXTERIOR que por limitaciones espaciales no se presenta en la figura. El desarrollo del tesauro en clases jerárquicas es uno de los aspectos que sustenta un software, evita que se repita un mismo término en diferentes categorías y obliga a escoger una, utilizando otro tipo de relación como la asociativa (Jacob, 2004: 89).

El desarrollo de los árboles de dominio de cada uno de los espacios ayuda a estudiar, analizar y conocer el ámbito en detalle, pero como se aprecia en los ejemplos anteriores, la estructura del tesauro es mucho más que eso. "Cuando se observa un objeto se busca identificar los rasgos comunes que tiene con otros objetos que se conocen, se abstraen algunos de los elementos que lo componen para identificar similitudes" (Naumis Peña, 2000: 98).

El siguiente árbol de dominio elaborado fueron los auditorios. Sin du$\mathrm{da}$, los auditorios como espacios culturales no cuentan con un desarrollo tan amplio de las áreas en que se distribuyen los términos del teatro, pero existe coincidencia en algunos aspectos menores que también se presentan en los auditorios.

Por ejemplo, la programación en los auditorios exige plasmar con claridad la estructura del área administrativa que gestiona los espacios en cada dependencia o las posibilidades del área técnica para acceder desde el exterior directamente al escenario, porque en general en el escenario de los auditorios no se contempla la comunicación exterior, la existencia de un vestíbulo ni tampoco una plaza de acceso donde se pueda programar un brindis.

Al estudiar la posición del AUDITORIO como descriptor se transformó y eliminó SALA DE TEATRO, porque la sala es una característica de los espacios donde se junta el público para asistir a un evento y por tanto se repite en todos los espacios culturales. Por esta razón el término preferido fue SALA y la relación asociativa del término recuerda a quien va a utilizarlo que debe asociarlo con la categoría espacial que corresponda: teatro, auditorio, cine, etc. Siempre la inclusión de un término tiene que balancear una solución que oscila entre el uso del término por los hablantes y qué propósito está cumpliendo (Kwaśnik, 2019: 664). 
Para finalizar la etapa de aprendizaje, al integrar los términos en el software MultiTes Pro se unificaron los árboles de dominio en uno solo. Se establecieron dos categorías generales: áreas y atributos y recursos técnicos, que a su vez se interrelacionan con la categoría de espacios culturales para unificar el universo de la ICU. Al conformar los tipos de espacios en el tesauro, por ejemplo, con TEATRO, el esquema en su desarrollo jerárquico y asociativo se transformó como se observa en la Figura 2.

\section{INFRAESTRUCTURA CULTURAL UNIVERSITARIA}

\section{ESPACIOS CULTURALES}

TE

BIBLIOTECA

CINE

EXPLANADA

FORO AL AIRE LIBRE

MUSEO

SALA DE CONCIERTOS

SALA DE CONFERENCIAS

SALA DE PROYECCIÓN

AUDIOVISUAL

SALA DE USOS MÚLTIPLES

SALÓN DE DANZA

SALÓN DE MÚSICA

TEATRO

TE

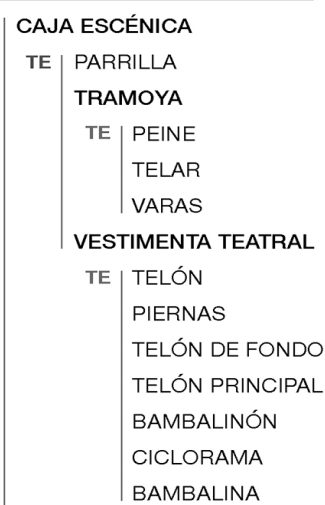

DISCO GIRATORIO

FOSO DE ORQUESTA

FOYER

OFICINAS DEL TEATRO

PASO DE GATO

TRAMPILLAS

\section{ÁREAS Y ATRIBUTOS}

TE | ÁREA ESCÉNICA

TE | DESAHOGOS

ESCENARIO

FORO

PROSCENIO

RETROESCENARIO

ÁREA PÚBLICA EXTERIOR

TE $\mid$ ESTACIONAMIENTO

PLAZA DE ACCESO

ÁREA PÚBLICA INTERIOR

TE | CAFETERÍA

CIRCULACIÓN VERTICAL

PAQUETERIA

PATIO

RESTAURANTE

SALA

TE | PASILLO

ESCALERA DE SALA

RAMPA DE SALA

BUTACAS

TE | BUTACAS NUMERADAS

TE | FILAS

GRADERIAS

ACCESO A ESCENARIO

ACCESO A SALA

TE | ACCESO PARA DISCAPACITADOS

ESPACIOS RESERVADOS PARA DISCAPACITADOS EN SALA

PLATEA

PLATEA SALA

SANITARIOS PÚBLICOS

TAQUILLA

TERRAZA

Figura 2. Estructura jerárquica y asociativa del espacio TEATRO en el tesauro integrado con todas las categorías espaciales, exceptuando los recursos técnicos

Fuente: adaptación Luis E. Sánchez 
La etapa final de evaluación del resultado obtenido con este experimento de trabajo colectivo está siendo realizada por la CDC y los diferentes especialistas en la programación de actividades culturales en la universidad. Los especialistas en el tema serán quienes validen la autoridad que emana de los términos escogidos para representar el ámbito de práctica y obtener un producto intelectual de calidad, capaz de responder a las necesidades de las comunidades interesadas. La experiencia de integrar a los alumnos fue un éxito porque aprendieron activamente, experimentando y resolviendo los ejercicios en la práctica.

\section{CONSIDERACIONES FINALES}

La discusión del ámbito de conocimiento para construir el sistema de información es una oportunidad de aprendizaje para los estudiantes participantes en el proyecto. Los dos estudiantes de Arquitectura que aplicaron los cuestionarios e hicieron los levantamientos en los espacios concluyeron sus tesis y presentaron sus exámenes profesionales; se están elaborando una tesis de doctorado y tres de licenciatura en Bibliotecología basadas en la experiencia directa con la elaboración del tesauro.

El tesauro es el producto de una investigación sobre cada unidad de conocimiento significativa y su función en el conjunto de los elementos que conforman un sistema de información. El primer paso es el desarrollo de un árbol de dominio de la especialidad, partiendo de cada una de las categorías principales que agrupan tales conocimientos de acuerdo con sus elementos comunes, los que a la vez separan, con base en otros elementos que establecen las diferencias.

La operación de construcción y mantenimiento de un tesauro es de un alto costo en investigación y desarrollo, que redunda en beneficio para organizar la información del sistema, comunicarse entre especialistas de diferentes ámbitos y también en la indización y recuperación sobre las búsquedas realizadas en la base de datos.

En los tesauros se conectan por su significado descriptores y términos equivalentes y descriptores con descriptores, de modo tal que uno nos conduzca a los otros con rigurosidad en la reciprocidad. El término tiene una función descriptiva en un ámbito de conocimiento que sirve de base a la construcción del descriptor para adquirir una función activa y dinámica a través del contexto, que lo ayuda a significar para el sistema donde será insertado. 
El Sistema de Información para el Registro Universitario de Espacios y Activos

Culturales (SI-RUEyAC) es un proyecto universitario financiado por el Programa de Apoyo a Proyectos de Investigación e Innovación Tecnológica (PAPIT), y forma

parte del proyecto apoyado con recursos PAPIIT IT400318, respaldado por la Dirección General de Asuntos del Personal Académico de la Universidad Nacional Autónoma de México (UNAM). Participan en él estudiantes de Arquitectura, Bibliotecología y Lengua y Literaturas Modernas Inglesas, así como personal de la Coordinación de Difusión Cultural (CDC) e investigadores del Instituto de Investigaciones Bibliotecológicas y de la Información (IIBI).

Colaboradores por área: Estructura y relación entre árboles de dominio, Hugo Alberto Guadarrama Sánchez y Luis Enrique Sánchez Rodríguez; Manejo del software: Hugo Alberto Guadarrama Sánchez; Áreas y atributos: Hugo Alberto Guadarrama Sánchez y Luis Enrique Sánchez Rodríguez; Espacios culturales: Hugo Alberto Guadarrama Sánchez, Luis Enrique Sánchez Rodríguez, Rosa de Guadalupe Hernández Villeda, Jimena Furlong Pineda, Brenda Espinoza Ledesma y Alondra Sarai Sánchez Laguna; Recursos técnicos: Hugo Alberto Guadarrama Sánchez y Luis Enrique Sánchez Rodríguez; Coordinador del SI-RUEyAC, Andrés Ramírez Aguirre.

\section{REFERENCIAS}

Baskerville, Richard L. y A. Trevor Wood-Harper. 1996. "A Critical Perspective on Action Research as a Method for Information Systems Research". Journal of Information Technology (11): 235-246. https://doi.org/10.1177/026839629601100305

Barité, Mario. 2019. "Towards a General Conception of Warrants: First Notes". Knowledge Organization 46 (8): 647-655. https://doi.org/10.5771/0943-7444-2019-8-647

Campos, Maria Luiza de Almeida, Maria Luiza Machado Campos, Hagar Espanha Gomes, Linair Maria Campos, Alissandra Evangelista Martins y Luana Farias Salas. 2006. "Estudo comparativo de softwares de construção de tesauros". Perspectivas em Ciência da Informação 11 (1): 68-81. https://doi.org/ 10.1590/S1413-99362006000100006

Clavier, Viviane. 2019. "Data and Algorithms: The New Era of Visual Representation”. Knowledge Organization 46 (8): 615-621. https://doi.org/10.5771/0943-7444-2019-8-615

Fugmann, Robert. 1992. "Indexing Quality - Predictability versus Consistency”. International Classification 19 (1): 20-21.

Fugmann, Robert. 1993. Subject Analysis and Indexing: Theoretical Foundations and Practical Advice. Frankfurt/Main: Index Verlag.

Getty Research Institute. 2019. Art and Architecture Thesaurus Online (AAT). http://www.getty.edu/research/tools/vocabularies/aat/about.html 
Hjørland, Birger. 2002a. "Domain analysis in information science: eleven approaches-traditional as well as innovative". Journal of Documentation 58 (4): 422-462. https://doi.org/10.1108/00220410210431136

Hjørland, Birger. 2002b. "Epistemology and the Socio-Cognitive Perspective in Information Science". Journal of the American Society for Information Science and Technology 53(4): 257-270. https://doi.org/10.1002/asi.10042

Hjørland, Birger. 2015. "Are Relations in Thesauri 'Context Free, Definitional, and True in all Possible Worlds'?” Journal of the Association for Information Science and Technology 66 (7): 1367-1373.

https://doi.org/10.1002/asi.23253

ISO 25964. 2011. 2013. The International Standard for Thesauri and Interoperability with other vocabularies. Part 1 Thesauri and Information retrieval (2011) Part 2 Interoperability with other vocabularies (2013). http://www.niso.org/schemas/iso25964/

Jacob, Elin. 2004. "The structure of context: implications of structure for the creation of context in information systems". Advances in Knowledge Organization 9: 85-90. https://www.ergon-verlag.de/isko_ko/downloads/aikovol09200415.pdf

Kwaśnik, Barbara H. 2019. "Changing Perspectives on Classification as a Knowledge-Representation Process”. Knowledge Organization 46 (8): 656-667. https://doi.org/10.5771/0943-7444-2019-8-656

Lykke, Marianne. 2001. "A framework for work task-based thesaurus design". Journal of Documentation 57 (6): 774-797. https://doi.org/10.1108/EUM0000000007100

Martínez Tamayo, Ana M. y Paola V. Mendes. 2015. Diseño y desarrollo de tesauros. La Plata: Universidad Nacional de la Plata, Facultad de Humanidades y Ciencias de la Educación.

Naumis Peña, Catalina. 2000. "Análisis de la confluencia entre término y descriptor en la elaboración de tesauros". Investigación Bibliotecológica: archivonomía, bibliotecología e información 14 (29): 95-113.

Naumis Peña, Catalina. 2003. "Indización y clasificación: un problema conceptual y terminológico". Documentación de las Ciencias de la Información 26: 23-40.

Naumis Peña, Catalina, Ariel A. Rodríguez García y Hugo Alberto Guadarrama Sánchez. 2019. "Una terminología de teatro para organizar el patrimonio cultural universitario”. Trabajo presentado en el IV Congreso ISKO España-Portugal (XIV Congreso ISKO España). Barcelona, 11- 12 de julio. http://eprints.rclis.org/38511/

RAE (Real Academia Española). 2019. Diccionario de la Lengua Española, s.v. "infraestructura". https://dle.rae.es/infraestructura?m=\&e

Smiraglia, Richard. P. 2012. "Epistemology of Domain Analysis". In Cultural Frames of knowledge, edited by Richard P. Smiraglia and Hur-Li Lee, 111-124. Würzburg: Ergon-Verlag.

Theatre Projects Consultants. 2016. Parts of a theatre building. http://theatreprojects.com/files/pdf/partsofatheatrebuilding.pdf

Wikipedia. 2019, s.v. "Centro Cultural Universitario (UNAM)". Fecha de consulta: 25 de noviembre de 2019. https://es.wikipedia.org/wiki/Centro_Cultural_Universitario_(UNAM) 
EL TESAURO DE INFRAESTRUCTURA CULTURAL UNIVERSITARIA...

Para citar este texto:

Naumis-Peña, Catalina. 2020. "El tesauro de infraestructura cultural universitaria: investigación y metodología”. Investigación Bibliotecológica: archivonomía, bibliotecología e información 34 (85): 137-153. http://dx.doi.org/10.22201/iibi.24488321xe.2020.85.58229 